\title{
FDA Quality Metrics Terminology
}

National Cancer Institute

\section{Source}

National Cancer Institute. FDA Quality Metrics Terminology. NCI Thesaurus. Code C123273.

Terminology developed to support activities regarding quality metrics within the FDA operations. 